\title{
Compreensão de informações em saúde pelos idosos atendidos no SUS estimulados pela ludicidade
}

\author{
Playfulness promoted understanding of health \\ information by elderly attended in the SUS
}

\author{
Maria Izabel Penha de Oliveira Santos', Andresa Pereira Silva', \\ Nubia Caroline Carvalho Rego' \\ 'Universidade do Estado do Pará, Grupo de Ensino, Pesquisa e Extensão sobre a Saúde do Idoso da Amazônia, \\ Belém (PA), Brasil.
}

\section{RESUMO}

Objetivo: Comparar a capacidade de compreensão de informações em saúde de um grupo de idosos através da estimulação com jogos educativos. Método: Pesquisa quantitativa, de cunho descritivo e comparativo, realizada com 40 idosos assistidos em Unidade Municipal de Saúde em Belém/PA. Foram compostos dois grupos: um Grupo Lúdico (GL) com n=20 e outro Grupo Não Lúdico (GNL) com n=20. Os dados foram coletados em três etapas: (1) Aplicação de um formulário estruturado contendo dados sociodemográficos e de saúde, (2) leitura de um texto sobre Hipertensão Arterial seguida de (3) avaliação da compreensão textual e numérica, com os dois grupos. Na segunda etapa o GL participou de um jogo para a estimulação da memória e concentração, e na terceira etapa os dois grupos fizeram a releitura do texto e reavaliação da compreensão textual e numérica. Resultados: Dentre os idosos, 55,0\% eram do sexo feminino, a média de idade foi 70 anos (DP $\pm 7,24$ ); a média de escolaridade foi de 6 e 8 anos de estudo, para o GNL e GL, respectivamente (DP \pm 4,06 e DP $\pm 3,95$ ); cerca de $75,0 \%$ relataram ter hábito de leitura e 70,0\% referiram compreender as informações em saúde, nos dois grupos. Quanto à compreensão textual, o resultado da primeira avaliação foi semelhante, $65,0 \%$ para o GNL e 70,0\% para GL $(p>0,05)$. Conclusão: Conforme a primeira avaliação textual e numérica houve uma boa compreensão dos grupos. Com a utilização dos jogos educativos, o GL apresentou proporcionalmente melhor compreensão textual em relação ao GNL. Assim, observou-se que os dois grupos de idosos compreenderam de forma semelhante às informações textuais e numéricas, contribuindo, possivelmente, para os cuidados em saúde.

\section{COMO CITAR ESTE ARTIGO}

Santos MIPO, Silva AP, Rego NCC

Compreensão de informações em

saúde pelos idosos atendidos no SUS estimulados pela ludicidade. Interdisciplinary Journal of Health Education. 2016 Jan-Jul:1(1):40-48. http://dx.doi.org/10.4322/ijhe2016004

palavras-chave: Alfabetização em saúde. Idoso. Saúde do Idoso

\section{ABSTRACT}

Aim: To compare the ability to understand health information by a group of elderly using stimulating educational games. Material and Methods: Quantitative research of descriptive and comparative nature, performed with 40 elderly assisted in the Municipal Health Unit in Belem/PA. Two groups were formed: one Ludic Group (LG) with $n=20$ and a Non-ludic Group (NLG) with $n=20$. Data were collected in three steps: (1) Application of a structured questionnaire containing demographic and health data, (2) reading of a text on hypertension followed by (3) evaluation of textual and numerical understanding with both groups combined. In the second step, the LG participated in a game for stimulating memory and concentration, and in the third step, the two groups made a rereading of the text and reevaluation of textual and numerical understanding. Results: Among the elderly, $55.0 \%$ were female, the average age was 70 years (SD \pm 7.24 ); the average education was 6 and 8 years of study for NLG and LG, respectively (SD \pm 4.06 and SD \pm 3.95 ); about $75.0 \%$ reported reading habit and $70.0 \%$ reported understand health information in both groups. Regarding comprehension, results of the first assessment was similar for NLG $65.0 \%$ and $70.0 \%$ for LG ( $p>0.05$ ). Conclusion: According to the first textual and numerical evaluation, the groups showed good understanding. With the use of educational games, LG had proportionally better reading comprehension in relation to NLG. Thus, it was observed that the two groups of elderly understood similarly to the textual and numerical information, possibly contributing to the health care.

KEYWORDS: Health literacy. Elderly. Health elderly. 


\section{Introdução}

De acordo com a Organização Mundial de Saúde, em 2025 o número de idosos com 60 anos ou mais, passará para aproximadamente 32 milhões de pessoas. Atualmente existe no Brasil, cerca de 20 milhões de pessoas com idade igual ou superior a 60 anos, o que significa pelo menos $10 \%$ da população brasileira ${ }^{1}$.

Dessa forma, o poder público, preocupado com essa demanda, através de leis de proteção social, o Estatuto do Idoso $^{2}$, art. 21, prevê a criação, de oportunidades de acesso do idoso à educação, adequando currículos, metodologias e material didático aos programas educacionais a ele destinados. Além disso, existe também a preocupação pelos profissionais de saúde, de como esses idosos estão informados e compreendem a sua própria saúde e consequentemente ao seu autocuidado.

A capacidade de compreensão de informações em saúde é também chamada de alfabetização em saúde ou letramento funcional em saúde, que pode ser definidos como o grau de aptidão das pessoas para adquirir, entender e dividir informações em saúde, ou seja, são aspectos relacionados aos cuidados em saúde, com a finalidade de preservar e estimular a sua promoção ao decorrer do ciclo vital ${ }^{3,4}$.

Sobre esse aspecto, estudo revelou que indivíduos com adequada alfabetização em saúde ou letramento funcional em saúde em comparação aos que possuem esse aspecto inadequado tendem a apresentar menor adesão a tratamentos, medicamentos, habilidades de autocuidado, irregularidades nas visitas médicas, déficit no controle das doenças e qualidade de vida, custos médicos elevados e maiores taxas de hospitalização e mortalidade ${ }^{5}$.

Nesse âmbito, foi evidenciada em uma revisão integrativa da literatura realizada no período de 2005 a 2014 sobre o tema, que na literatura nacional na área de enfermagem, apenas dois estudos foram publicados, em comparação com quinze encontrados na literatura internacional no mesmo período. Sobre os estudos nacionais, foram realizados por pesquisadores da Região Sul, sinalizando que a temática além de ser pouco discutida, ainda e pouco explorada no Brasil e em especial na Região Norte ${ }^{6}$.

Dessa forma, essa possível lacuna na ciência e sobretudo na área da enfermagem gerontológica inquietou as autoras a realizar este estudo, que traçaram como objetivo principal: avaliar a capacidade de compreensão de informações em saúde de um grupo de idosos através da estimulação com jogos educativos.

\section{Materiais e métodos}

Trata-se de uma pesquisa quantitativa, de cunho observacional, descritivo e comparativo, com $(\mathrm{N}=40)$ idosos, de ambos os sexos assistidos por uma Unidade Municipal de Saúde em Belém. Foram selecionados por conveniência dois grupos de idosos cada um com $(n=20)$. A seleção dos idosos foi baseada na população de idosos que participava de alguma atividade lúdica/ou de lazer, ofertada pela Unidade de Saúde, então a partir dessa referência alocaram-se os idosos nos grupos. Esses grupos foram denominados: Grupo lúdico (GL), cujos participantes foram os idosos que desenvolviam alguma atividade lúdica/recreativa desenvolvidas na referida Unidade e na mesma proporção, o Grupo Não Lúdico (GNL), composto por aqueles que não participavam de tais atividades. Os critérios para participar do estudo eram saber ler e escrever, e se conseguiam ler um texto sobre saúde que continha informações textuais e numéricas como o parâmetros a pressão arterial normal em adultos. Foram excluídos, idosos com diagnóstico médico de distúrbio mental ou analfabetos.

Os dados foram coletados em três etapas: Na primeira etapa participaram os dois grupos informando sobre as variáveis sociodemográficas e da saúde. Nessa mesma 
etapa, os idosos dos dois grupos eram convidados a ler um texto sobre a Hipertensão Arterial, adaptado do manual da Secretaria Estadual de Saúde, que versava sobre os fatores de risco, medidas preventivas, controle e parâmetros da pressão arterial fisiológica. Após a leitura do texto, os idosos foram avaliados quanto à compreensão textual e numérica através de um questionário, que permitia apenas uma resposta.

A segunda etapa consistiu na participação apenas do GL em dois jogos educativos, com o objetivo de estimular a concentração e memória. Ao término, foi avaliado também o desempenho dos idosos nesses jogos (tempo gasto jogo, compreensão do objetivo do jogo e dificuldades para jogar) através de um questionário próprio que continha uma escala dicotômica de compreensão ou não compreensão. No que se refere às variáveis: tempo gasto no jogo, esse aspecto era observado pelos pesquisadores e registrado no instrumento impresso, além disso, as possíveis dificuldades encontradas pelos idosos, estas foram informadas pelos participantes e depois categorizadas como: não houve, concentração, grande número de peças e tempo que poderiam ter sido variáveis que implicariam na capacidade de concentração e entendimento do texto.

Ainda sobre os jogos, foram selecionados dois jogos com peças de objetos como meio de transportes, em que o examinado teria que identificar o par do objeto, nesse caso, continha vinte peças. O outro jogo era formado pelas parte do corpo humano e o examinado deveria encaixar as partes desse corpo no local adequado.

Na terceira etapa da coleta de dados participaram os dois grupos de idosos, que releram o texto e foram reavaliados, referentes à sua compreensão textual e numérica.

As informações coletadas foram organizadas e agrupadas em um banco de dados no Programa Statistical Package for the Social Science (SPSS), versão 20.0. Os resultados foram descritos em proporções e comparados entre o grupo Lúdico (GL) e não Lúdico (GNL), pelas medidas de tendência central (media, mediana e desvio padrão) e Teste Exato de Fisher, estabelecendo-se o nível $\alpha=5 \%$ e p valor $\leq 0,05$.

Destaca-se que esta pesquisa respeitou os fundamentos éticos estabelecidos na Resolução 466/12. O projeto foi submetido ao Comitê de Ética e Pesquisa (CEP) da Universidade do Estado do Pará, com parecer aprovado $N^{\circ} 1.360 .765$, sendo utilizado o Termo de Consentimento Livre Esclarecido para ambos os grupos.

\section{Resultados}

De acordo com os resultados apresentados na Tabela 1, observa-se que a média de idade foi semelhante entre os dois grupos, assim como, a faixa etária de 60-70 anos foi a mais prevalente também para os dois grupos. A maior proporção foi de mulheres em comparação aos homens do estudo, a média de anos de estudo entre os idosos variou de 6 a 8, a maior proporção relatou ter hábito de leitura e participação social e lazer.

De acordo com a Tabela 2 a maior proporção de idosos apresentava comorbidades (mais de duas doenças associadas). Observa-se que os idosos de ambos os grupos faziam algum tipo de tratamento médico, assim como o uso de medicação. Mais da metade dos idosos, de ambos os grupos referiram possuir esquecimento habitual, e a compreensão de informações em saúde.

De acordo com a Tabela 3, observa-se que na compreensão textual da primeira leitura, os grupos apresentaram compreensão semelhantes, assim como, cerca de $60,0 \%$ dos idosos compreenderam o sentido do texto e mais de $85,0 \%$, as informações numéricas.

Observa-se na Tabela 4, que a maior proporção de idosos que participaram da atividade lúdica, mais de 95,0\%, compreenderam os objetivos dos jogos de memória utilizados. O tempo de desempenho dos jogos variou entre 11 a 20 minutos, nos dois grupos. No primeiro jogo, metade dos participantes considerou seu desempenho 
Tabela 1. Perfil sociodemográfico dos idosos que participaram do estudo. Belém/PA, $2016(n=40)$.

\begin{tabular}{|c|c|c|c|c|}
\hline Variáveis & \multicolumn{2}{|c|}{$\begin{array}{c}\text { Grupo Não Lúdico } \\
\text { (GNL) } \\
\text { f \% }\end{array}$} & \multicolumn{2}{|c|}{$\begin{array}{c}\text { Grupo Lúdico (GL) } \\
\text { f \% }\end{array}$} \\
\hline \multicolumn{5}{|l|}{ Idade (anos) } \\
\hline \multicolumn{5}{|l|}{ GNL (Média = 70; DP $\pm 7,24$ ) } \\
\hline \multicolumn{5}{|l|}{ GL (Média= 69,2; DP $\pm 5,59$ ) } \\
\hline \multicolumn{5}{|l|}{ Faixa etária } \\
\hline $60-70$ & 11 & 55,0 & 12 & 60,0 \\
\hline $71-80$ & 7 & 35,0 & 8 & 40,0 \\
\hline $81-+$ & 2 & 10,0 & 0 & 0,0 \\
\hline \multicolumn{5}{|l|}{ Sexo } \\
\hline Feminino & 11 & 55,0 & 18 & 90,0 \\
\hline Masculino & 9 & 45,0 & 2 & 10,0 \\
\hline \multicolumn{5}{|l|}{ Escolaridade } \\
\hline \multicolumn{5}{|l|}{ GNL (Média= 6,20; DP \pm 4,06) } \\
\hline \multicolumn{5}{|l|}{ GL (Média =7,90; DP $\pm 3,95$ ) } \\
\hline \multicolumn{5}{|l|}{ Escolaridade (anos) } \\
\hline $1-4$ & 10 & 50,0 & 6 & 30,0 \\
\hline $5-9$ & 4 & 20,0 & 7 & 35,0 \\
\hline $10-12$ & 4 & 20,0 & 5 & 25,0 \\
\hline $13-18$ & 2 & 10,0 & 2 & 10,0 \\
\hline \multicolumn{5}{|l|}{ Hábito de leitura* } \\
\hline Sim & 17 & 85,0 & 15 & 75,0 \\
\hline Não & 3 & 15,0 & 5 & 25,0 \\
\hline \multicolumn{5}{|l|}{ Participação social e lazer** } \\
\hline Sim & 9 & 45,0 & 20 & 100,0 \\
\hline Não & 11 & 55,0 & 0 & 00,0 \\
\hline
\end{tabular}

Nota: *Hábito de leitura (revistas, livros religiosos e mais de um tipo de livro); **Participação social (Dança, caminhada, musculação, hidroginástica, teatro).

Tabela 2. Condições de Saúde dos idosos que participaram do estudo. Belém/PA, $2016(n=40)$.

\begin{tabular}{|c|c|c|c|c|}
\hline Variáveis & \multicolumn{2}{|c|}{$\begin{array}{c}\text { Grupo Não Lúdico } \\
\text { (GNL) }\end{array}$} & \multicolumn{2}{|c|}{ Grupo Lúdico (GL) } \\
\hline \multicolumn{5}{|l|}{ Doenças prevalentes } \\
\hline Comorbidades & 8 & 40,0 & 15 & 75,0 \\
\hline Hipertensão Arterial & 11 & 55,0 & 8 & 40,0 \\
\hline Colesterol alto & 3 & 15,0 & 1 & 5,0 \\
\hline Lombalgia & 0 & 00,0 & 1 & 5,0 \\
\hline Outras & 1 & 05,0 & 1 & 5,0 \\
\hline Nenhuma & 4 & 20,0 & 2 & 10,0 \\
\hline \multicolumn{5}{|l|}{ Tratamento médico } \\
\hline Sim & 18 & 90,0 & 14 & 70,0 \\
\hline Não & 2 & 10,0 & 6 & 30,0 \\
\hline \multicolumn{5}{|l|}{ Uso de medicação } \\
\hline Sim & 18 & 90,0 & 16 & 80,0 \\
\hline Não & 2 & 10,0 & 4 & 20,0 \\
\hline \multicolumn{5}{|l|}{ Esquecimento } \\
\hline Sim & 11 & 55,0 & 12 & 60,0 \\
\hline Não & 9 & 45,0 & 8 & 40,0 \\
\hline \multicolumn{5}{|c|}{ Compreensão de informações em saúde } \\
\hline Sim & 14 & 70,0 & 14 & 70,0 \\
\hline Não & 6 & 30,0 & 6 & 30,0 \\
\hline
\end{tabular}


Tabela 3. Comparação da capacidade de compreensão de informações textuais e numéricas dos idosos que participaram do estudo. Belém/PA, $2016(n=40)$.

\begin{tabular}{|c|c|c|c|c|}
\hline \multirow{2}{*}{ Variávei } & \multicolumn{2}{|c|}{ Grupo Não Lúdico (GNL) } & \multicolumn{2}{|c|}{ Grupo Lúdico (GL) } \\
\hline & \multicolumn{2}{|c|}{$f \%$} & \multicolumn{2}{|c|}{$f \%$} \\
\hline \multicolumn{5}{|c|}{ Compreensão textual $\mathrm{p}=0,277>0,05$} \\
\hline Sim & 12 & 60,0 & 14 & 70,0 \\
\hline Não & 8 & 40,0 & 6 & 30,0 \\
\hline \multicolumn{5}{|c|}{ Compreensão numérica } \\
\hline Sim & 17 & 85,0 & 18 & 90,0 \\
\hline Não & 3 & 15,0 & 2 & 10,0 \\
\hline
\end{tabular}

Nota: Textual= texto sobre a Hipertensão Arterial (o que fala o texto; riscos de ter pressão arterial, métodos preventivos); numérica (valor da pressão arterial normal). Teste Exato de Fisher.

Tabela 4. Capacidade de compreensão e desempenho dos idosos que participaram da atividade lúdica (GL) do estudo. Belém/PA, $2016(n=20)$.

\begin{tabular}{|c|c|c|c|c|}
\hline Variáveis & \multicolumn{2}{|c|}{ Jogo 1} & & \\
\hline Sim & 19 & 95,0 & 20 & 100,0 \\
\hline Não & 1 & 05,0 & 0 & 0,0 \\
\hline \multicolumn{5}{|c|}{ Tempo de desempenho do Jogo (min) } \\
\hline \multicolumn{5}{|c|}{ Jogo 1 (Média=25,50s; DP $\pm 21,16 \mathrm{~s}$ ) } \\
\hline \multicolumn{5}{|c|}{ Jogo 2 (Média=16,10s; DP $\pm 8,71 s$ ) } \\
\hline \multicolumn{5}{|c|}{ Tempo de desempenho do Jogo (min) } \\
\hline $1-10$ & 4 & 20,0 & 6 & 30,0 \\
\hline $11-20$ & 8 & 40,0 & 7 & 35,0 \\
\hline $21-30$ & 4 & 20,0 & 7 & 35,0 \\
\hline $31-+$ & 4 & 20,0 & 0 & 0,0 \\
\hline \multicolumn{5}{|l|}{ Percepção do desempenho } \\
\hline Bom & 10 & 50,0 & 16 & 80,0 \\
\hline Regular & 6 & 30,0 & 3 & 15,0 \\
\hline Excelente & 4 & 20,0 & 1 & 5,0 \\
\hline \multicolumn{5}{|l|}{ Dificuldade no jogo } \\
\hline Concentração & 9 & 45,0 & 5 & 25,0 \\
\hline Lembrar onde estava a peça & 8 & 40,0 & 13 & 65,0 \\
\hline Grande número de peças & 1 & 05,0 & 1 & 5,0 \\
\hline Tempo & 0 & 00,0 & 1 & 5,0 \\
\hline Não houve & 2 & 10,0 & 0 & 0,0 \\
\hline
\end{tabular}

Nota: Os jogos 1 e 2 foram escolhidos aleatoriamente pelos idosos. (memórias educativas, meios de transporte e comunicação e conheça o corpo humano).

bom, no segundo jogo a proporção aumentou, chegando a 80,0\%. As dificuldades mais relatadas foram à concentração e a sequência do jogo.

Conforme os resultados apresentados na Tabela 5, à compreensão textual foram semelhantes antes e após a atividade lúdica. Em relação à numérica permaneceu a mesma. 
Tabela 5. Comparação da capacidade de compreensão de informações textuais e numéricas do Grupo Lúdico (GL) antes e após a atividade lúdica dos idosos que participaram do estudo. Belém/PA, 2016 $(n=20)$.

\begin{tabular}{|c|c|c|c|c|}
\hline \multirow{2}{*}{ Variáveis } & \multicolumn{2}{|c|}{ Antes da AL } & \multicolumn{2}{|c|}{ Depois da AL } \\
\hline & \multicolumn{2}{|c|}{$f \%$} & \multicolumn{2}{|c|}{$f \%$} \\
\hline Compreensão textual & & & & $\mathrm{p}=0,61>0,05$ \\
\hline Sim & 14 & 70,0 & 16 & 80,0 \\
\hline Não & 6 & 30,0 & 4 & 20,0 \\
\hline \multicolumn{5}{|l|}{ Compreensão numérica } \\
\hline Sim & 18 & 90,0 & 18 & 90,0 \\
\hline Não & 2 & 10,0 & 2 & 10,0 \\
\hline
\end{tabular}

Nota: Textual= texto sobre a Hipertensão Arterial (o que fala o texto; riscos de ter pressão arterial, métodos preventivos); numérica (valor da pressão arterial normal). Teste Exato de Fisher.

\section{Discussão}

\section{Aspectos sociodemográficos e da saúde dos idosos do estudo}

A média de idade dos idosos que compõem o estudo comportou-se como a média geral de idade dos brasileiros. Os resultados indicaram maior participação feminina, que pode estar relacionada: a sua maior longevidade; maior procura pelos serviços médicos, e percepção mais efetiva dos riscos à saúde que os homens, pois possuem mais acesso às informações. A média de anos de estudo também foram mais elevadas que a média nacional, que corresponde a 4,8 anos de estudo ${ }^{7-10}$.

Os resultados referentes à leitura convergem com outro estudo ${ }^{11}$, que considera ser relevante para elevar os ganhos cognitivos, como a memória. Além disso, a prática de atividades sociais vivenciada pelos idosos do estudo também está acima da média nacional, na qual apenas $13,6 \%$ dos idosos tem acesso ao esporte e lazer ${ }^{12}$.

A presença associada de doenças (comorbidades) nos idosos, bem como da Hipertensão Arterial Sistêmica também pode ser verificado em outros estudos ${ }^{13,14}$. Destaca-se que a maior frequência de doenças associadas foi encontrada no GL, do qual todos fazem atividade física. A prática de exercício físico funciona como um tratamento não farmacológico para doenças crônicas, capaz de reduzir níveis tensionais, excesso de peso, resistência à insulina e dislipidemias ${ }^{15}$.

Além disso, o processo de envelhecimento populacional provoca uma elevação da utilização dos serviços de saúde, devido a maior prevalência, entre os idosos, de doenças e incapacidades, assim como a maior vulnerabilidade biológica ${ }^{16}$, o que corrobora a presença de algum tipo de tratamento médico entre os idosos, o que pode ser observado neste estudo, principalmente do GNL. O acentuado uso de fármacos, e a prevalência na utilização de anti-hipertensivos, também foram encontrados em outros estudos $^{17-19}$. O menor uso de fármacos utilizado pelo GL pode ser justificado pela utilização de medidas alternativas não farmacológicas, como a atividade física ${ }^{15,20}$.

O esquecimento habitual referido pelos dos dois grupos, está relacionado a possíveis perdas cognitivas, que pode ocorrer mesmo no processo normal de envelhecimento, que pode variar de esquecimentos benignos até casos de demência ${ }^{21}$.

Compreensão textual e numérica do texto sobre hipertensão arterial pelos idosos do estudo

No presente estudo, conforme os resultados houve uma compreensão textual e numérica semelhante entre os dois grupos, embora maior proporcionalmente no GL. Sobre a compreensão textual e numérica de uma informação, convergindo com outros autores ${ }^{22}$, discute-se que, o sentido de um texto é construído na interação 
texto e sujeito. A leitura é uma atividade com elevado grau de produção de sentido. Ela requer um enorme conjunto de saberes, além de, não ser um simples produto de código e letras exige do leitor um conhecimento prévio. Neste caso vale destacar que mais da metade dos idosos tinham ou tratavam HAS, podendo ter contribuído para esse resultado.

A compreensão de um texto e/ou a sua interpretação por um indivíduo requer que ele tenha além da alfabetização formal aprendido na escola, que inclui leitura e escrita, mas também um nível de letramento capaz de fazê-lo entender as dimensões de sua vida ${ }^{23}$. Desse modo, é muito comum na área da saúde se ofertar materiais didáticos sobre saúde, sobretudo de prevenção de doenças, porém não se avalia se esses materiais didáticos de fato alfabetizam a pessoa na área da saúde, ou seja, se lhe permitem interpretar de que forma essas informações vão ser aplicadas no seu dia-a-dia e consequentemente como podem impactar no autocuidado. Assim, embora alfabetização e letramento recebam conotações diferentes, porém são indissociáveis do ponto de vista teórico.

Sobre a compreensão numérica o GL apresentou maior proporção quando comparado ao GNL, esse resultado pode ter sido influenciado pelo nível de escolaridade. Vale ressaltar que bons resultados de compreensão de informações de saúde estão intimamente ligados com altos níveis de escolaridade. E, isso pode ser explicado quando se avalia o letramento funcional em saúde, uma vez que índices de escolaridades baixos estão relacionados aos piores cuidados de saúde ${ }^{6}$. Nesse sentido, idosos que tem dificuldades no cuidado com sua saúde ao longo e médio prazo podem tornar-se dependente de cuidados de outrem.

Além disso, um estudo relacionando compreensão numérica com a versão do guia alimentar para a população geral brasileira, afirma que para o leitor entender a relação numérica é necessário o conhecimento prévio ${ }^{24}$. Os autores deste estudo utilizam como exemplo o Índice de Massa Corporal (IMC), assim, para o entendimento, o leitor deve possuir um conhecimento prévio do significado da relação entre o peso e a altura; de conceitos científicos/ matemáticos, tal qual calorias, valor calórico, quantidade média, equivalências entre porções de alimentos, dentre outros. No presente estudo, a compreensão numérica era o valor da pressão arterial que estava em uma proporção, ou seja, para os idosos para entender necessitavam de um conhecimento prévio sobre essa habilidade.

A autopercepção de desempenho nos jogos educativos tem caráter subjetivo, pois cada um se percebe de um modo diferente, e pode sofrer influencia de fatores como humor e pressão social ${ }^{25}$. Muito embora, não tenha sido objeto deste estudo, mas observou-se empiricamente, que os idosos do GL sentiram-se agraciados por ter tido oportunidade de participar de jogos de estimulação de memória; expressaram nas suas faces contentamento, bom estado de humor e felicidade subjetiva, animação, participação e sorrisos constantes durante a execução da atividade lúdica. Percebeu-se também o despertar para a diversão e estimulação mutua.

Sobre esse aspecto lúdico na atenção em saúde, como a utilização dos jogos de memória, permite-se o estimulo a atenção, percepção, raciocínio e pensamento dos idosos. Todavia, para resultados mais efetivos é necessário um processo longo e continuo de atividades, com grau de complexidade crescente ${ }^{26,27}$.

A compreensão textual do GL nos dois momentos de leitura, observou-se que houve uma diferença mínima, em torno de 5,0\%, porém, não se podendo afirmar se a atividade lúdica melhorou o desempenho deste grupo na leitura. Esse resultado pode estar ligado ao tempo limitado para o desenvolvimento deste estudo, que não permitiu o acompanhamento por um período maior. Pois, em outros estudos, houve um resultado satisfatório na aplicação de jogos na alfabetização em saúde, porém o estudo realizou oficinas, no qual idosos afirmaram que o programa de estimulação da memória contribuiu para melhora da memória nas atividades cotidianas ${ }^{28}$. 


\section{Limitações do estudo}

Uma das limitações do presente estudo foi à utilização de uma amostra pequena $(\mathrm{n}=40)$, não permitindo possíveis generalizações. Além disso, só foi possível a aplicação de apenas dois jogos educativos, devido o tempo limitado para execução do estudo, mas que acredita-se que proporcionou uma estimulação mínima da memória, sendo necessários maiores sessões para um resultado mais efetivo. Outra limitação foi à utilização de um tema para a avaliação da compreensão textual e numérica já conhecida pelos idosos (Hipertensão Arterial), o que possivelmente, permitiu a utilização de conhecimentos anteriores à leitura do texto.

\section{Conclusões}

Os idosos avaliados neste estudo referiram compreender as informações em saúde, o que talvez seja um fator positivo para seu autocuidado. A capacidade de compreensão textual e numérica avaliada tanto para o grupo que participou da atividade lúdica quanto para os que não participaram apresentou-se semelhante na primeira e na segunda avaliação. Muito embora, o grupo que não participou da atividade lúdica (os dois jogos), cerca de 45,0\%, participava de algum tipo de atividade de participação social, ou seja, eram ativos, o que talvez tenha influenciado nesse resultado.

\section{Referências}

1. Brasil. Ministério da Saúde. Departamento de Ações Programáticas e Estratégicas. Atenção à saúde da pessoa idosa e envelhecimento. Área Técnica Saúde do Idoso. Brasília; 2010.

2. Brasil. Presidência da República, Casa Civil, Subchefia para Assuntos Jurídicos. Lei $n^{\circ} 10.741$ de $1^{\circ}$ de Outubro de 2003. Dispõe sobre o Estatuto do Idoso e dá outras providências. Diário Oficial da União [Internet], Brasília, DF, 3 out. 2003 [citado em 2016 Maio 12]. Disponível em: http://www.planalto.gov.br/ccivil_03/leis/2003/L10.741.htm

3. Paskulin LMG, Aires M, Valer DB, Morais EP, Freitas IVA. Adaptação de um instrumento que avalia alfabetização em saúde das pessoas idosas. Acta Paul Enferm. 2011;24(2):271-277. http://dx.doi.org/10.1590/S0103-21002011000200018.

4. Paskulin LMG, Bierhals CCB, Valer DB, et al. Alfabetização em saúde de pessoas idosas na atenção básica. Acta Paul Enferm. 2012;25:129-35. http://dx.doi.org/10.1590/S0103-21002012000800020.

5. Santos LTM, Mansur HN, Paiva TFPS, Colugnati FAB, Bastos MG. Letramento em Saúde: importância da avaliação em nefrologia. J Bras Nefrol. 2012;34(3):293-302. PMid:23099838. http://dx.doi.org/10.5935/0101-2800.20120014.

6. Santos MIPO, Porte"1la MR, Scortegagna HM, Santos PCS. Letramento funcional em saúde na perspectiva da Enfermagem Gerontológica: revisão integrativa da literatura. Rev Bras Geriatr Gerontol. 2015;18(3):651-64. http://dx.doi. org/10.1590/1809-9823.2015.14080.

7. Instituto Brasileiro de Geografia e Estatística - IBGE. Ministério do Planejamento, Orçamento e Gestão. Coordenação de População e Indicadores Sociais. Diretoria de Pesquisas. Síntese de indicadores sociais: uma analise das condições de vida da população brasileira [Internet]. Brasília; 2012 [citado em 2016 Maio 12]. Disponível em: http://biblioteca.ibge.gov.br/ visualizacao/livros/liv66777.pdf

8. Camargos MCS, Gonzaga MR. Viver mais e melhor? Estimativas de expectativa de vida saudável para a população brasileira. Cad Saude Publica. 2015;31(7):1460-72. PMid:26248101. http://dx.doi.org/10.1590/0102-311X00128914.

9. Pilger C, Menon MU, Mathias TAF. Utilização de serviços de saúde por idosos vivendo na comunidade. Rev Esc Enferm. 2013;47(1):213-20. http://dx.doi.org/10.1590/S0080-62342013000100027.

10. Dias FA, Tavares DMS. Fatores associados à participação de idosos em atividades educativas grupais. Rev Gaucha Enferm. 2013;34(2):70-7. PMid:24015464. http://dx.doi.org/10.1590/S1983-14472013000200009.

11. Torquato R, Massi G, Santana AP. Envelhecimento e letramento: a leitura e a escrita na perspectiva de pessoas com mais de 60 anos de idade. Psicol Reflex Crit. 2011;24(1):89-98. http://dx.doi.org/10.1590/S0102-79722011000100011.

12. Instituto Brasileiro de Geografia e Estatística-IBGE. Coordenação de População e Indicadores Sociais. Síntese de indicadores sociais: uma análise das condições de vida da população brasileira: 2015. Rio de Janeiro: IBGE; 2015 . 137 p. Estudos e Pesquisas. Informação demográfica e socioeconômica, 35.

13. Nunes DP, Nakatani AYK, Silveira EA, Bachion MM, Souza MR. Capacidade funcional, condições socioeconômicas e de saúde de idosos atendidos por equipes de Saúde da Família de Goiânia (GO, Brasil). Cien Saude Colet. 2010;15(6):2887-98. PMid:20922297. http://dx.doi.org/10.1590/S1413-81232010000600026.

14. Ferreira PCS, Tavares DMS, Rodrigues RAP. Características sociodemográficas, capacidade funcional e morbidades entre idosos com e sem declínio cognitivo. Acta Paul Enferm. 2011;24(1):29-35.

15. Moraes WM, Souza PRM, Pinheiro MHNP, Irigoyen MC, Medeiros A, Koike MK. Programa de exercícios físicos baseado em frequência semanal mínima: efeitos na pressão arterial e aptidão física em idosos hipertensos. Rev Bras Fisioter. 2011;16(2):1-8. 


\section{ijhe}

16. Virtuoso JF, Mazo GZ, Menezes EC, Cardoso AS, Dias RG, Balbé GP. Perfil de morbidade referida e padrão de acesso a serviços de saúde por idosos praticantes de atividade física. Cien Saude Colet. 2012;17(1):23-31. PMid:22218536. http:// dx.doi.org/10.1590/S1413-81232012000100005.

17. Silva CSO, Pereira MI, Yoshitome AY, Rodrigues JF No, Barbosa DA. Avaliação do uso de medicamentos pela população idosa em Montes Claros, Minas Gerais, Brasil. Esc Anna Nery. 2010;14(4):811-8. http://dx.doi.org/10.1590/S1414-81452010000400022.

18. Pizzol TSD, Pons ES, Hugo FN, Bozzetti MC, Sousa MLR, Hilgert JB. Uso de medicamentos entre idosos residentes em áreas urbanas e rurais de municípios no Sul do Brasil: um estudo de base populacional. Cad Saude Publica. 2012;28(1):10414. PMid:22267070. http://dx.doi.org/10.1590/S0102-311X2012000100011.

19. Paiva SCL, Gomes CP, Almeida LG, et al. A influencia das comorbidades, do uso de medicamentos e da institucionalização na capacidade funcional dos idosos. Rev Int Est Exp 2014;6:46-53.

20. Secoli SR. Polifarmácia: interações e reações adversas no uso de medicamentos por idosos. Rev Bras Enferm. 2010;63(1):13640. PMid:20339769. http://dx.doi.org/10.1590/S0034-71672010000100023.

21. Oliveira CS, Costa SRR, Santos ICL, Lemos CES. Oficina de educação, memória, esquecimento e jogos lúdicos para a Terceira Idade. Rev Ciênc Ext. 2012;8(1):8-17.

22. Koch IV, Elias VM. Ler e compreender: Os sentidos do texto. 3. ed. São Paulo: Contexto; 2013.

23. Soares M. Letramento: um tema em três gêneros. 3. ed. Belo Horizonte: Autentica; 2012.

24. Passamai MPB, Sampaio EAC, Dias AMI, Cabral LA. Letramento funcional em saúde: reflexões e conceitos sobre seu impacto na interação entre usuários, profissionais e sistema de saúde. Interf Comunic Saude Educ. 2011;16(41):301-14. http://dx.doi.org/10.1590/S1414-32832012005000027.

25. Andradea NB, Canon MBF, Zugman CL, Ayres TG, Ide MG, Novelli MMPC. Centro de convivência de idosos: uma abordagem de estimulação cognitiva e psicossocial. Cad Ter Ocup. 2014;22(1):121-8.

26. Santos IB, Gomes L, Matos NM, et al. Oficinas de estimulação cognitiva adaptadas para idosos analfabetos com transtorno cognitivo leve. Rev Bras Enferm. 2012;65(6):962-8. PMid:23559175. http://dx.doi.org/10.1590/S0034-71672012000600012.

27. Araújo PO, Silveira EC, Bôas AMV, Silva JD. Promoção da saúde do idoso: a importância do treino da memória. Rev Kair Geront. 2012;15(8):169-83.

28. Sato AT, Batista MP, Almeida MHM. Programas de estimulação da memória e funções cognitivas relacionadas: opiniões e comportamentos dos idosos participantes. Rev Ter Ocup Univ Sao Paulo. 2014;25(1):51-9. http://dx.doi.org/10.11606/ issn.2238-6149.v25i1p51-59.

\section{Contribuição dos autores}

Maria Izabel Penha de Oliveira Santos participou da concepção, da coleta, análise dos dados e elaboração do artigo. Andresa Pereira Silva e Nubia Caroline Carvalho Rego auxiliaram a coleta, a análise dos dados e elaboração do artigo. 